\title{
COLPOSCOPIC REFERRAL FOR REPEATED INADEQUATE SMEARS: IS IT NECESSARY?
}

\author{
MRA Said, Staff Grade, Obstetrics \& Gynaecology;
}

\section{BA Teague, Manager, E Stewart, Assistant, Clinical Audit Furness General Hospital}

\section{INTRODUCTION}

Between five and ten per cent of all smears are inadequate. Taking adequate smears will remove the anxiety induced when women are asked to return for a repeat smear, as well as reduce the overall workload for practices. Also, in terms of the GP contract, the calculations of targets are based on adequate smear tests only.

A cervical smear, if properly taken, should contain cells from the whole of the transformation zone, which should therefore be adequately sampled. Squamous epithelial cells will normally be the most numerous cell type. The main evidence of an adequate smear is that it should contain a sufficient quantity of epithelial cells, taking into account a woman's age and her hormonal status. Properly taken cervical smears are more likely to be achieved in the right surroundings, ie with privacy, good lighting, and a relaxed patient.

An indication that the transformation zone has been properly sampled is the additional presence of endocervical columnar cells and recognizable metaplastic cells. Endocervical cells may not always be seen in smears from post-menopausal women, or those with atrophic smears.

If a smear is inadequate the woman will need to have it repeated. She should be informed why the repeat smear is required, so as to allay the anxiety that this is likely to cause her.

An audit was carried out to determine whether it is necessary to refer patients who have had two or more inadequate smears to the colposcopy clinic.

\section{OBJECTIVES}

The objectives were to determine

- incidence of colposcopic referral for inadequate smears

- age group of patients who had repeated inadequate smears

- associated symptoms

- colposcopic findings

- histology

- associated wart virus

- management

- follow-up

\section{MATERIALS AND METHODS}

The colposcopy clinic diaries were used as the data source to identify patients who attended the colposcopy clinic during the period 1 January to 30 June 1999. One hundred and fortyseven patients were identified and their case notes were extracted for review. A proforma was designed for retrospective data collection. Information was entered into EPI Info V4.0b, a World Health Organisation database and statistical package.

\section{RESULTS}

Thirty-seven of the 147 patients $(25 \%)$ who were referred for colposcopy between 1 January and 30 June 1999 were referred for repeated inadequate smears. The age distribution of patients referred for inadequate smears is shown in Figure 1.

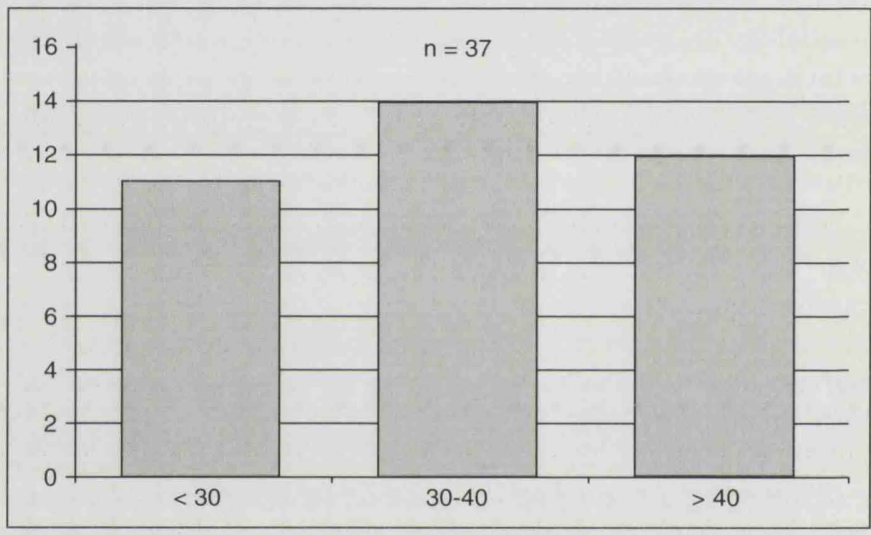

Figure 1 Age distribution

There were four patients among those who were referred for inadequate smear who had previous treatment for abnormal smears. Two of the four patients had cold coagulation, one had hysterectomy, and one had laser treatment elsewhere. Thirty patients (81\%) were asymptomatic, while two patients were complaining of abnormal vaginal discharge. Three patients presented with post-coital bleeding, one patient with inter-menstrual bleeding and one patient with post-menopausal bleeding. Nine patients (24\%) were regular cigarette smokers.

Colposcopic examination revealed that 25 patients $(68 \%)$ were normal, while 12 patients (32\%) had abnormal colposcopic findings, such as acetowhite epithelium or mosaic appearance.

Thirty-four patients had a repeat smear upon their first visit to the colposcopy clinic. The results of this test are shown in Table 1.

\begin{tabular}{|c|c|c|}
\hline SMEAR RESULT & NUMBER & $\%$ \\
\hline negative smear & 31 & $91 \%$ \\
\hline mild dyskariosis & 1 & $3 \%$ \\
\hline inadequate & 2 & $6 \%$ \\
\hline
\end{tabular}

Table 1 Smear results taken at colposcopy clinic 
Thirty patients had an additional colposcopically-directed punch biopsy of the cervix. The results are shown in Table 2.

\begin{tabular}{|l|c|c|}
\hline HISTOLOGY RESULT & NUMBER & $\%$ \\
\hline normal & 19 & $63.3 \%$ \\
\hline inflammatory & 4 & $13.3 \%$ \\
\hline CIN1 & 2 & $6.7 \%$ \\
\hline HPV & 4 & $13.3 \%$ \\
\hline insufficient for diagnosis & 1 & $3.3 \%$ \\
\hline
\end{tabular}

Table 2 Histology of cervical punch biopsy

\section{MANAGEMENT}

Twenty-five patients $(68 \%)$ were discharged following the results of repeated smear and histology of cervical punch biopsy. Four patients $(10.8 \%)$ required cold coagulation and two $(5.4 \%)$ had loop diathermy excision of the transformation zone. Six patients $(16.2 \%)$ required repeated follow-up smear (Table 3).

\begin{tabular}{|l|c|c|}
\hline MANAGEMENT & NUMBER & $\%$ \\
\hline discharged & 25 & $67.6 \%$ \\
\hline cold coagulation & 4 & $10.8 \%$ \\
\hline loop excision & 2 & $5.4 \%$ \\
\hline follow-up smear & 6 & $16.2 \%$ \\
\hline
\end{tabular}

Table 3 Management of patients referred with inadequate smear

\section{DISCUSSION}

Nearly $25 \%$ of the overall workload of the colposcopy clinic at Furness General Hospital is directed towards patients referred with repeated inadequate smears. The main evidence of an adequate smear is a sufficient quantity of epithelial cells. Endocervical cells do not necessarily have to be present for the smear to be adequate and, unless otherwise advised, the next smear should be carried out at the normal screening interval.

Reasons for failure of the cervical smear test are:

- patient is very tense owing to insufficient reassurance

- cervix is not visualized adequately

- cervix is not scraped firmly enough

- transformation zone is not completely scraped

- material is incompletely transferred to the slide

- sample is poorly spread (too thick, or too thin, or distorted due to excessive pressure)

- smear is allowed to dry before fixation

- insufficient fixative is used

- smear consists mainly of blood or inflammatory cell exudate, possibly associated with menstruation

- there is contamination of the smear with lubricant, vaginal cream, or spermicide

- menstrual smears contain large numbers of endometrial cells

A brush as well as a spatula should be considered in the following conditions:

- where there is difficulty in inserting the spatula into the cervical os

- when the last smear was inadequate due to absence of transformation zone sampling
- when the woman has a history of CIN2 or 3 and follow-up smears have lacked transformation zone sampling

- when a woman has previously been treated for cervical adenocarcinoma

The position of the transformation zone varies. The part of the transformation zone adjacent to the squamo-columnar junction (SCJ) is the most vulnerable to CIN. When the SCJ is visible, the sample must include the whole circumference of the SCJ and the adjacent $1 \mathrm{~cm}$ of squamous epithelium. When the SCJ is in the canal and not visible, a sampler must be chosen that will remove cells from the canal, in addition to the ectocervix.

Copious cervical mucus can remove the cells from the sampler as it is withdrawn, resulting in a reduction in the number of cells on the slide. Gentle removal of the discharge away from the os with the blunt end of the sampler may help to overcome such problems.

The national standard for inadequate smears in any one laboratory is $7 \% \pm 2 \%$. The national standard for an individual smear taker is that at least $80 \%$ of smears taken from women aged 20-50 years contain evidence of sampling from the transformation zone, ie contain metaplastic and/or endocervical cells.

The majority (91\%) of smear results taken at the colposcopy clinic, in patients referred for inadequate smear, were negative. This may reflect unnecessary referral to colposcopy clinics.

Only two out of 37 patients (5\%) had low-grade lesions (CIN 1) which required diathermy loop excision under local anaesthetic. Four patients had cold coagulation for associated symptoms, such as post-coital bleeding, inter-menstrual bleeding, or persistent vaginal discharge.

\section{CONCLUSION}

Inadequate smears represent a significant volume $(25 \%)$ of the workload of patients referred to the colposcopy clinic at Furness General Hospital. Taking adequate smears will help to reduce the overall workload for the colposcopy clinic and remove the anxiety induced when women are asked to visit such a clinic.

\section{RECOMMENDATIONS}

Smear takers need to have an annual record of the number of adequate and inadequate smears they have taken.

Identification of individuals with unacceptably high inadequate rates will enable them to undergo further training. Continuous monitoring of these inadequate rates can be used to measure levels of performance amongst all smear takers, but is particularly useful for new smear takers, or those who have required retraining.

\section{BIBLIOGRAPHY}

BSCC, Taking cervical smears. 1989 p18

McPherson, Cervical screening: a practical guide. 1st edition, OUP, 1985

NHS Cervical Screening programme, Resource Pack for Training Smear Takers, NHSCSP Publication No 9, 1998 